\title{
Atomic properties of superheavy elements No, Lr, and Rf
}

\author{
V. A. Dzuba \\ School of Physics, University of New South Wales, Sydney, 2052, Australia \\ M. S. Safronova \\ Department of Physics and Astronomy, University of Delaware, Newark, Delaware, USA and \\ Joint Quantum Institute, NIST and the University of Maryland, College Park, Maryland, USA \\ U. I. Safronova \\ Physics Department, University of Nevada, Reno, Nevada, USA
}

\begin{abstract}
The combination of the configuration interaction method and all-order single-double coupledcluster technique is used to calculate excitation energies, ionization potentials and static dipole polarizabilities of superheavy elements nobelium, lawrencium and rutherfordium. Breit and quantum electrodynamic corrections are also included. The results for the superheavy elements are compared with earlier calculations where available. Similar calculations for lighter analogs, ytterbium, lutetium, and hafnium are used to study the accuracy of the calculations. The estimated uncertainties of the final results are discussed.
\end{abstract}

PACS numbers: 31.15.vj, 31.30.jg, 11.30.Er

\section{INTRODUCTION}

The study of the superheavy elements (nuclear charge $Z>100)$ is an important multidisciplinary area of research involving nuclear physics, atomic physics, and chemistry (see, e.g. reviews [1-3]). Atomic calculations help to understand the role of the relativistic and manybody effects and provide important information for the planing and interpreting the measurements. The need to treat relativistic and correlation effects to high level of accuracy makes the calculations a very challenging task. Relativistic effects are most important for the structure of the inner electron shells. Their effect on the spectra of neutral atom, determined by valence electrons, is much smaller. Standard approach based on using Dirac equation and adding Breit and quantum electrodynamic (QED) corrections gives reasonably good results (see, e.g. [4 7]). Accurate treatment of correlations is a more difficult task. Most of superheavy elements have open shells with many valence electrons and strong correlations between them and between valence electrons and electrons in the core. Therefore, it is particulary important to establish the benchmark values for superheavy systems that have one to four valence electrons which can be treated by the most high-precision approaches. Such calculations also establish the importance of various corrections that may be used for more complicated superheavy atoms. In our previous papers [4 7] we studied the elements with nuclear charge $Z=112,119$ and 120 , which are heavier analogs of mercury, francium and radium respectively. These systems have one or two valence electrons. In present paper, we calculate the spectra and other atomic properties of superheavy atoms with two, three, and four valence electrons above closed shells: nobelium $(Z=102)$, lawrencium $(Z=103)$, and rutherfordium $(Z=104)$. These elements are heavier analogs of ytterbium, lutetium and hafnium. No, Lr, and Rf were studied theoretically in [8 $[17]$ and experimentally in [1 3], but experimental spectra still have not been measured. Present relativistic calculation use the combination of the configuration interaction (CI) method with the linearized single-double coupled cluster method (CI+SD or CI+all-order) 18]. Correlations between valence electrons are treated with the CI technique while correlations between core and valence electrons are included via single-double coupled-cluster method. This approach provides the most complete treatment of the inter-electron correlations since it includes core-core, core-valence and valence-valence correlations to all orders. We treat nobelium, lawrencium and rutherfordium as two-, three-, and four-valence electrons systems respectively. Previous calculations for No $[8-10]$ and Rf [16, 17] considered these atoms as two-valence electron systems, while calculations for $\operatorname{Lr}[11-15]$ treated the atom as a monovalent system. Such treatments omit important correlation effects for Lr and Rf. Comparing present and earlier calculations provide important information on the role of different types of correlation and relativistic corrections. We also present calculations of few first ionization potentials for No, Lr and Rf, up to removal of all valence electrons and calculate static polarizabilities for all three atoms. In next section we describe the method and present results of calculations for $\mathrm{Yb}, \mathrm{Lu}$, and $\mathrm{Hf}$ to illustrate the accuracy of the method. In last section we present results and detailed discussion for No, Lr, and Rf.

\section{METHOD OF CALCULATION}

The calculations are performed using the configuration interaction method combined with the linearized singledouble coupled-cluster method introduced in [18]. This 
CI+all-order method yielded accurate atomic properties for a number of divalent systems and trivalent Tl [1821]. It has been recently applied to the calculations of four-electron systems for the first time (Sn-like ions) [22].

We use frozen core Dirac-Fock (DF) $V^{N-M}$ potential [23] as the point of departure for all of our calculations, where $N$ is the total number of electrons and $M$ is the number of valence electrons, i.e. the initial DF procedure is carried out for the closed-shell ion, with all valence electrons removed. For the atoms treated here, $M=2$ for $\mathrm{Yb}$ and No, $M=3$ for $\mathrm{Lu}$ and $\mathrm{Lr}$, and $M=4$ for Hf and Rf. The effective CI Hamiltonian for states of valence electrons is the sum of single-electron Hamiltonians and an operator representing the interaction between the valence electrons,

$$
\hat{H}^{\mathrm{eff}}=\sum_{i=1}^{M} \hat{h}_{1}\left(r_{i}\right)+\sum_{i<j} \hat{h}_{2}\left(r_{i}, r_{j}\right)
$$

The single-electron Hamiltonian for a valence electron has the form

$$
\hat{h}_{1}=c \alpha \mathbf{p}+(\beta-1) m c^{2}-\frac{Z e^{2}}{r}+V^{N-M}+\hat{\Sigma}_{1},
$$

where $\hat{\Sigma}_{1}$ is the correlation potential operator, which represents the correlation interaction of a valence electron with the core. Its matrix elements are related to the single-excitation amplitudes of the coupled-cluster method via

$$
\Sigma_{m v}=\rho_{m v}\left(\widetilde{\epsilon}_{v}-\epsilon_{m}\right)
$$

where $\rho_{m v}$ is an excitation coefficient of the atomic wave function for the term with excitation from the valence state $v$ to another excited state $m$; and $\epsilon_{m}$ are Dirac-Fock energies of corresponding single-electron basis states. The quantities $\widetilde{\epsilon}_{v}$ are discussed in detail in Ref. [18]. Briefly, the CI+all-order approach is based on the Brillouin-Wigner variant of MBPT rather than the Rayleigh-Schrödinger variant resulting in the energy dependence of the $\Sigma$. Ideally, the energy $\widetilde{\epsilon}_{v}$ should be calculated from the particular eigenvalue of the effective Hamiltonian. In actual calculations, the simplest and the most practical approach is to set the energy $\widetilde{\epsilon}_{v}$ to the Dirac-Fock energy of the lowest orbital for the particular partial wave. For example, we use $\widetilde{\epsilon}_{v}=\epsilon_{6 s}$ for all $n s$ orbitals of $\mathrm{Yb}$ atom.

The interaction between valence electrons is the sum of the Coulomb interaction and the correlation correction operator $\hat{\Sigma}_{2}$ :

$$
\hat{h}_{2}\left(r_{i}, r_{j}\right)=\frac{e^{2}}{\left|\mathbf{r}_{\mathbf{i}}-\mathbf{r}_{\mathbf{j}}\right|}+\hat{\Sigma}_{2}\left(r_{i}, r_{j}\right)
$$

The operator $\hat{\Sigma}_{2}$ represents the screening of the Coulomb interaction between valence electrons by core electrons. Its matrix elements are related to the double-excitation coupled-cluster $\rho_{\text {mnvw }}$ coefficients via

$$
\Sigma_{m n v w}=\rho_{m n v w}\left(\widetilde{\epsilon}_{v}+\widetilde{\epsilon}_{w}-\epsilon_{m}-\epsilon_{n}\right) .
$$

The many-electron wave function for the valence electrons $\Psi$ can be expressed as an expansion over singledeterminant wave functions

$$
\Psi=\sum_{i} c_{i} \Phi_{i}\left(r_{1}, \ldots, r_{M}\right) .
$$

The functions $\Phi_{i}$ are constructed from the single-electron valence basis states calculated in the $V^{N-M}$ potential. The coefficients $c_{i}$ and many-electron energies are found by solving the matrix eigenvalue problem

$$
\left(H^{\mathrm{eff}}-E\right) X=0
$$

where $H_{i j}^{\text {eff }}=\left\langle\Phi_{i}\left|\hat{H}^{\text {eff }}\right| \Phi_{j}\right\rangle$ and $X=\left\{c_{1}, c_{2}, \ldots, c_{n}\right\}$.

We use the linearized coupled-cluster method to calculate the correlation correction operators $\hat{\Sigma}_{1}$ and $\hat{\Sigma}_{2}$. The B-spline technique [24] is used to construct a singleelectron basis for calculation of $\hat{\Sigma}$ and for building manyelectron basis states for the CI calculations. We use 35 $B$-splines of order 7 in a cavity of radius $R_{\max }=60 a_{B}$, where $a_{B}$ is Bohr's radius. All sums in the all-order terms are carried out including $l_{\max }=6$ partial waves. The contributions from $l>6$ partial waves was estimated and included into the final results.

\section{A. Breit interaction}

Breit interaction is included in present calculations using the approach developed in Ref. [25, 26]. We treat Breit interaction in zero energy transfer approximation. The Breit Hamiltonian includes magnetic interaction between moving electrons and retardation

$$
\hat{H}^{B}=-\frac{\boldsymbol{\alpha}_{1} \cdot \boldsymbol{\alpha}_{2}+\left(\boldsymbol{\alpha}_{1} \cdot \mathbf{n}\right)\left(\boldsymbol{\alpha}_{2} \cdot \mathbf{n}\right)}{2 r} .
$$

Here $\mathbf{r}=\mathbf{n} r, r$ is the distance between electrons, and $\boldsymbol{\alpha}$ is the Dirac matrix.

Similar to the way Coulomb interaction is used to form self-consistent Coulomb potential, Breit interaction is used to form self-consistent Breit potential. In other words, Breit interaction is included into self-consistent Hartree-Fock procedure. Thus the important relaxation effect is included. The resulting inter-electron potential in (2) consist of two terms

$$
\hat{V}=V^{C}+V^{B}
$$

$V^{C}$ is the Coulomb potential, $V^{B}$ is the Breit potential. Coulomb interaction in the second-order correlation operator $\hat{\Sigma}$ is also modified to include Breit operator (8). The contribution of the Breit interaction to the energy levels of all atoms considered here is small, generally less than $100 \mathrm{~cm}^{-1}$.

\section{B. QED corrections}

We use the radiative potential method developed in Ref. [27] to include quantum radiative corrections. This 
potential has the form

$$
V_{\mathrm{rad}}(r)=V_{U}(r)+V_{g}(r)+V_{e}(r)
$$

where $V_{U}$ is the Uehling potential, $V_{g}$ is the potential arising from the magnetic formfactor, and $V_{e}$ is the potential arising from the electric formfactor. The $V_{U}$ and $V_{e}$ terms can be considered as additions to nuclear potential while inclusion of $V_{g}$ leads to some modification of the Dirac equation (see Ref. [27] for details). We find that the QED corrections are small in comparison with the higher-order correlation corrections and can be omitted at the present level of accuracy. We compared the results with and without QED for No as an illustration.

\section{Calculation of polarizabilities}

Polarizabilities characterize interaction of atoms with external electric field. The Stark energy shift of atomic state $J L n$ in the static electric field $\varepsilon$ is given by

$$
\Delta E(J L n)=-\left(\alpha_{0}+\frac{3 M^{2}-J(J+1)}{J(2 J-1)} \alpha_{2}\right) \frac{\varepsilon^{2}}{2},
$$

where $\alpha_{0}$ and $\alpha_{2}$ are scalar and tensor electric-dipole polarizabilities, and $M$ is the projection of the total angular momentum $J$ on the direction of electric field. Scalar polarizability is given by

$$
\alpha_{0}(J L n)=\frac{2}{3(2 J+1)} \sum_{J^{\prime} L^{\prime} n^{\prime}} \frac{\left\langle J L n\|\mathbf{D}\| J^{\prime} L^{\prime} n^{\prime}\right\rangle^{2}}{E_{n^{\prime}}-E_{n}},
$$

where $=-e \sum_{i} \mathbf{r}_{i}$ is the electric dipole operator. Tensor polarizability $\alpha_{2}$ is non-zero only for atomic states with $J \geq 1$. The expression for $\alpha_{2}$ differs from (12) by an angular coefficient:

$$
\begin{aligned}
& \alpha_{2}(J L n)=\sqrt{\frac{10 J(2 J-1)}{3(2 J+3)(2 J+1)(J+1)}} \times \\
& \sum(-1)^{\left(J+J^{\prime}\right)}\left\{\begin{array}{ccc}
1 & 1 & 2 \\
J & J & J^{\prime}
\end{array}\right\} \frac{\left\langle J L n\|\mathbf{D}\| J^{\prime} L^{\prime} n^{\prime}\right\rangle^{2}}{E_{n^{\prime}}-E_{n}}
\end{aligned}
$$

The expressions (12) and (13) are exact if $|J L n\rangle$ and $\left|J^{\prime} L^{\prime} n^{\prime}\right\rangle$ are exact many-electron wave functions. In practice, atomic electrons are divided into core and valence electrons and the expression for scalar polarizability becomes a sum of three terms

$$
\alpha_{0}=\alpha_{c}+\alpha_{c v}+\alpha_{v}
$$

Here $\alpha_{c}$ is the polarizability of atomic core, $\alpha_{c v}$ is the contribution caused by Pauli principle which implies that the excitations from the core cannot go into occupied valence states. Therefore, polarizability of the core is different for the ion, which has no valence electrons and for the neutral atom. This difference is separated into $\alpha_{c v}$. Usu-

\begin{tabular}{|c|c|c|c|c|c|c|c|}
\hline \multirow[t]{2}{*}{ Conf. } & \multirow[t]{2}{*}{ Term } & \multicolumn{3}{|c|}{ Energy } & \multicolumn{3}{|c|}{ g-factors } \\
\hline & & Expt. & Present & Diff. & Expt. & $\mathrm{nr}$ & Present \\
\hline $6 s^{2}$ & ${ }^{1} S_{0}$ & 0 & 0 & 0 & & 0 & 0 \\
\hline \multirow[t]{3}{*}{$6 s 6 p$} & ${ }^{3} P_{0}$ & 17288 & 17561 & -273 & & 0 & 0 \\
\hline & ${ }^{3} P_{1}$ & 17992 & 18261 & -269 & 1.49282 & 1.5000 & 1.4921 \\
\hline & ${ }^{3} P_{2}$ & 19710 & 20010 & -300 & 1.50 & 1.5000 & 1.5000 \\
\hline \multirow[t]{3}{*}{$5 d 6 s$} & ${ }^{3} D_{1}$ & 24489 & 24505 & -16 & 0.50 & 0.5000 & 0.5000 \\
\hline & ${ }^{3} D_{2}$ & 24752 & 24863 & -111 & 1.16 & 1.1667 & 1.1634 \\
\hline & ${ }^{3} D_{3}$ & 25271 & 25343 & -72 & 1.34 & 1.3333 & 1.3333 \\
\hline $6 s 6 p$ & ${ }^{1} P_{1}$ & 25068 & 25816 & -748 & 1.035 & 1.0000 & 1.0087 \\
\hline $5 d 6 s$ & ${ }^{1} D_{2}$ & 27678 & 27991 & -313 & 1.01 & 1.0000 & 1.0036 \\
\hline $6 s 7 s$ & ${ }^{3} S_{1}$ & 32695 & 32970 & -275 & 2.01 & 2.0000 & 1.9998 \\
\hline $6 s 7 s$ & ${ }^{1} S_{0}$ & 34351 & 34579 & -228 & & 0 & 0 \\
\hline \multirow[t]{3}{*}{$6 s 7 p$} & ${ }^{3} P_{0}$ & 38091 & 38377 & -286 & & 0 & 0 \\
\hline & ${ }^{3} P_{1}$ & 38174 & 38440 & -266 & 1.14 & 1.5000 & 1.4399 \\
\hline & ${ }^{3} P_{2}$ & 38552 & 38821 & -269 & 1.50 & 1.5000 & 1.4999 \\
\hline \multirow[t]{3}{*}{$6 s 6 d$} & ${ }^{3} D_{1}$ & 39809 & 40053 & -244 & 0.50 & 0.5000 & 0.5001 \\
\hline & ${ }^{3} D_{2}$ & 39838 & 40147 & -309 & 1.16 & 1.1667 & 1.1414 \\
\hline & ${ }^{3} D_{3}$ & 39966 & 40205 & -239 & 1.33 & 1.3333 & 1.3333 \\
\hline $6 s 6 d$ & ${ }^{1} D_{2}$ & 40062 & 40089 & -27 & 1.03 & 1.0000 & 1.1423 \\
\hline $6 s 7 p$ & ${ }^{1} P_{1}$ & 40564 & 39150 & 1414 & 1.01 & 1.0000 & 1.0598 \\
\hline $6 s 8 s$ & ${ }^{3} S_{1}$ & 41615 & 41997 & -382 & 2.02 & 2.0000 & 1.9994 \\
\hline $6 s 8 s$ & ${ }^{1} S_{0}$ & 41940 & 42397 & -457 & & 0 & 0 \\
\hline
\end{tabular}
ally this contribution is small and needs to be taken into
TABLE I: Energies $\left(E, \mathrm{~cm}^{-1}\right)$ [28] and $g$-factors of the lowest states of ytterbium. Comparison of calculations with experiment. Non-relativistic values of $g$-factors $\left(g_{\mathrm{nr}}\right)$ are given by (20).

${ }^{a}$ Ref. 29]

account only in very precise calculations. We neglect it in present work. The term $\alpha_{v}$ is the dominant contribution due to the valence electrons. The core contribution is given by

$$
\alpha_{c}=\frac{2}{3} \sum_{c m} \frac{\langle c m\|\mathbf{d}\| m\rangle\left\langle m\left\|\mathbf{d}+\delta \mathbf{V}_{\text {core }}\right\| c\right\rangle}{\epsilon_{c}-\epsilon_{m}}
$$

where summation goes over core states $c$ and a complete set of single-electron states $m$. The energies $\epsilon_{c}$ and $\epsilon_{m}$ are the single-electron energies of the basis states. The operator $\mathbf{d}=-e \mathbf{r}$ in (15) is the single-electron electric dipole operator, $\delta \mathbf{V}_{\text {core }}$ is the correction to the self-consistent core potential due to the effect of electric field. It is also known as the core polarization correction or random-phase approximation (RPA) correction. This correction is calculated by solving the RPA-type equations for atomic core

$$
\left(\hat{H}_{0}-\epsilon_{c}\right) \delta \psi_{c}=-\psi_{c}\left(\mathbf{d}+\delta \mathbf{V}_{\text {core }}\right),
$$

where $\hat{H}_{0}$ is the Hartree-Fock Hamiltonian, $\delta \psi_{c}$ is the correction to the core state $\psi_{c}$ due to the effect of external electric field. The equations (16) are solved selfconsistently for all states in the core and the correction 
TABLE II: Energies $\left(E, \mathrm{~cm}^{-1}\right)$ and $g$-factors of the lowest states of lutetium. Comparison of calculations with experiment. Non-relativistic values of $g$-factors $\left(g_{\mathrm{nr}}\right)$ are given by (20).

\begin{tabular}{|c|c|c|c|c|c|c|c|}
\hline \multirow[t]{2}{*}{ Conf. } & \multirow[t]{2}{*}{ Term } & \multicolumn{3}{|c|}{ Energy } & \multicolumn{3}{|c|}{$\mathrm{g}$-factors } \\
\hline & & Expt. & Present & Diff. & Expt. & $\mathrm{nr}$ & Present \\
\hline \multirow[t]{2}{*}{$5 d 6 s^{2}$} & ${ }^{2} D_{3 / 2}$ & 0 & 0 & 0 & 0.79921 & 0.8000 & 0.8000 \\
\hline & ${ }^{2} D_{5 / 2}$ & 1993 & 2014 & -21 & 1.20040 & 1.2000 & 1.1999 \\
\hline \multirow[t]{2}{*}{$6 s^{2} 6 p$} & ${ }^{2} P_{1 / 2}$ & 4136 & 3910 & 226 & 0.66 & 0.6666 & 0.6661 \\
\hline & ${ }^{2} P_{3 / 2}$ & 7476 & 7228 & 248 & 1.33 & 1.3333 & 1.3333 \\
\hline \multirow[t]{4}{*}{$5 d 6 s 6 p$} & ${ }^{4} F_{3 / 2}$ & 17427 & 17723 & -296 & 0.59 & 0.4000 & 0.4525 \\
\hline & ${ }^{4} F_{5 / 2}$ & 18504 & 18789 & -285 & 1.07 & 1.0286 & 1.0586 \\
\hline & ${ }^{4} F_{7 / 2}$ & 20432 & 20731 & -299 & 1.22 & 1.2381 & 1.2424 \\
\hline & ${ }^{4} F_{9 / 2}$ & 22609 & 22911 & -302 & 1.30 & 1.3333 & 1.3332 \\
\hline \multirow[t]{4}{*}{$5 d^{2} 6 s$} & ${ }^{4} F_{3 / 2}$ & 18851 & 19182 & -331 & & 0.4000 & 0.4109 \\
\hline & ${ }^{4} F_{5 / 2}$ & 19403 & 19737 & -334 & & 1.0286 & 1.0305 \\
\hline & ${ }^{4} F_{7 / 2}$ & 20247 & 20578 & -331 & & 1.2381 & 1.2368 \\
\hline & ${ }^{4} F_{9 / 2}$ & 21242 & 21591 & -349 & 1.0 & 1.3333 & 1.3313 \\
\hline \multirow[t]{4}{*}{$5 d 6 s 6 p$} & ${ }^{4} D_{1 / 2}$ & 20762 & 20995 & -233 & 0.00 & 0.0000 & 0.0353 \\
\hline & ${ }^{4} D_{3 / 2}$ & 21195 & 21448 & -253 & 1.19 & 1.2000 & 1.1551 \\
\hline & ${ }^{4} D_{5 / 2}$ & 22221 & 22504 & -283 & 1.39 & 1.3714 & 1.3799 \\
\hline & ${ }^{4} D_{7 / 2}$ & 23524 & 23795 & -271 & 1.41 & 1.4286 & 1.4171 \\
\hline \multirow[t]{2}{*}{$5 d 6 s 6 p$} & ${ }^{2} D_{5 / 2}$ & 21462 & 21735 & -273 & 1.23 & 1.2000 & 1.2107 \\
\hline & ${ }^{2} D_{3 / 2}$ & 22124 & 22376 & -252 & 0.874 & 0.8000 & 0.8591 \\
\hline \multirow[t]{3}{*}{$5 d^{2} 6 s$} & ${ }^{4} P_{1 / 2}$ & 21472 & 21860 & -388 & & 2.6667 & 2.6098 \\
\hline & ${ }^{4} P_{3 / 2}$ & 22467 & 22849 & -382 & 1.73 & 1.7333 & 1.7016 \\
\hline & ${ }^{4} P_{5 / 2}$ & 22802 & 23242 & -440 & & 1.6000 & 1.4749 \\
\hline \multirow[t]{3}{*}{$5 d 6 s 6 p$} & ${ }^{4} P_{1 / 2}$ & 24108 & 24520 & -412 & & 2.6667 & 2.6264 \\
\hline & ${ }^{4} P_{3 / 2}$ & 24308 & 24786 & -478 & 1.67 & 1.7333 & 1.6530 \\
\hline & ${ }^{4} P_{5 / 2}$ & 25191 & 25774 & -583 & 1.53 & 1.6000 & 1.5267 \\
\hline $5 d^{2} 6 s$ & ${ }^{2} D_{3 / 2}$ & 24518 & 25015 & -497 & & 0.8000 & 0.8379 \\
\hline
\end{tabular}

${ }^{a}$ Ref. [29]

to the core potential $\delta V_{\text {core }}$ is found. Core contribution is small, ranging from 3.20 a.u. for Hf to 8.46 a.u. for No. The core does not contribute to the tensor polarizability since the total angular momentum of the closed shell core is zero.

The expressions for the valence contributions to the scalar and tensor polarizabilities are very similar to (12) and (13) with a few modifications. The many-electron states $|J L n\rangle$ and $\left|J^{\prime} L^{\prime} n^{\prime}\right\rangle$ are now the valence states, the summation in the electric dipole operator $\mathbf{D}$ goes over only valence electrons, and every single-electron electric dipole operator $d$ is modified to include core polarization correction, $\tilde{\mathbf{d}}=\mathbf{d}+\delta \mathbf{V}_{\text {core }}$.

To perform summation in (12) and (13) over complete set of many-valence-electrons states we use method suggested by Dargarno and Lewis [31]. The summation is reduced to calculation of the correction to the ground state wave function

$$
\sum_{n} \frac{\langle a|| \tilde{D} \| n\rangle\langle n\|\tilde{D}\| a \mid\rangle}{E_{a}-E_{n}}=\langle a|| \tilde{D} \| \tilde{a}\rangle,
$$

TABLE III: Energies $\left(E, \mathrm{~cm}^{-1}\right)$ and $g$-factors of the lowest states of hafnium. Non-relativistic values of $g$-factors $\left(g_{\mathrm{nr}}\right)$ are given by Eq.20 Comparison of calculations with experiment [29]. Result with "*" is by Sansonetti and Martin [30].

\begin{tabular}{|c|c|c|c|c|c|c|c|}
\hline \multirow[t]{2}{*}{ Conf. } & \multirow[t]{2}{*}{ Term } & \multicolumn{3}{|c|}{ Energy } & \multicolumn{3}{|c|}{ g-factors } \\
\hline & & Expt. & Present & Diff. & Expt. & $\mathrm{nr}$ & Present \\
\hline \multirow[t]{3}{*}{$5 d^{2} 6 s^{2}$} & ${ }^{3} F_{2}$ & 0. & 0 & & 0.695 & 0.667 & 0.6936 \\
\hline & ${ }^{3} F_{3}$ & 2357 & 2343 & 14 & 1.083 & 1.083 & 1.0832 \\
\hline & ${ }^{3} F_{4}$ & 4568 & 4617 & -49 & 1.240 & 1.250 & 1.2425 \\
\hline \multirow[t]{3}{*}{$5 d^{2} 6 s^{2}$} & ${ }^{3} P_{0}$ & 5522 & 5611 & -89 & 0.00 & 0.00 & 0.00 \\
\hline & ${ }^{3} P_{1}$ & 6573 & 6594 & -21 & 1.500 & 1.500 & 1.5000 \\
\hline & ${ }^{3} P_{2}$ & 8984 & 9151 & -167 & 1.300 & 1.500 & 1.2783 \\
\hline $5 d^{2} 6 s^{2}$ & ${ }^{1} D_{2}$ & 5639 & 5842 & -203 & 1.165 & 1.000 & 1.1947 \\
\hline $5 d 6 s^{2} 6 p$ & ${ }^{1} D_{2}$ & $10509^{*}$ & 10095 & 414 & & 1.000 & 0.8173 \\
\hline $5 d^{2} 6 s^{2}$ & ${ }^{1} G_{4}$ & 10533 & 11411 & -878 & 1.008 & 1.000 & 1.0073 \\
\hline \multirow[t]{3}{*}{$5 d 6 s^{2} 6 p$} & ${ }^{3} D_{1}$ & 14018 & 13718 & 300 & 0.55 & 0.500 & 0.5384 \\
\hline & ${ }^{3} D_{2}$ & 16163 & 15840 & 323 & 1.17 & 1.167 & 1.1714 \\
\hline & ${ }^{3} D_{3}$ & 18381 & 18084 & 297 & 1.29 & 1.333 & 1.2980 \\
\hline \multirow[t]{4}{*}{$5 d^{3} 6 s$} & ${ }^{5} F_{1}$ & 14092 & 14445 & -353 & 0.00 & 0.00 & 0.0217 \\
\hline & ${ }^{5} F_{2}$ & 14741 & 15079 & -338 & 1.00 & 1.000 & 1.0038 \\
\hline & ${ }^{5} F_{3}$ & 15673 & 15996 & -323 & 1.25 & 1.250 & 1.2485 \\
\hline & ${ }^{5} F_{4}$ & 16767 & 17099 & -332 & 1.36 & 1.350 & 1.3445 \\
\hline \multirow[t]{3}{*}{$5 d 6 s^{2} 6 p$} & ${ }^{3} F_{2}$ & 14435 & 14019 & 416 & 0.89 & 0.666 & 0.8914 \\
\hline & ${ }^{3} F_{3}$ & 14542 & 14210 & 332 & 1.08 & 1.083 & 1.0877 \\
\hline & ${ }^{3} F_{4}$ & 18225 & 17887 & 338 & 1.24 & 1.250 & 1.2451 \\
\hline \multirow[t]{2}{*}{$5 d 6 s^{2} 6 p$} & ${ }^{3} P_{1}$ & 18143 & 17932 & 211 & 1.43 & 1.500 & 1.4401 \\
\hline & ${ }^{3} P_{2}$ & 19791 & 19584 & 207 & 1.41 & 1.500 & 1.4192 \\
\hline \multirow[t]{3}{*}{$5 d^{2} 6 s 6 p$} & ${ }^{5} G_{2}$ & 18011 & 17996 & 15 & 0.40 & 0.333 & 0.3874 \\
\hline & ${ }^{5} G_{3}$ & 19293 & 19262 & 31 & 0.95 & 0.917 & 0.9375 \\
\hline & ${ }^{5} G_{4}$ & 20960 & 20935 & 25 & 1.16 & 1.150 & 1.1597 \\
\hline
\end{tabular}

where correction $|\tilde{a}\rangle$ to the ground state wave function $|a\rangle$ is given by

$$
|\tilde{a}\rangle=\sum_{n}|b\rangle \frac{\langle a|| \tilde{D} \| n\rangle}{E_{a}-E_{n}} .
$$

The correction $|\tilde{a}\rangle$ satisfies the inhomogeneous equation

$$
\left(H^{\mathrm{CI}}-E_{a}\right)|\tilde{a}\rangle=-\tilde{D} H^{\mathrm{CI}}
$$

Here $H^{\mathrm{CI}}$ is the effective CI Hamiltonian presented in a matrix form while $|\tilde{a}\rangle$ is a vector of expansion coefficients over single-determinant basis states. Solving the system of linear equations (19) and substituting the result into (17) is equivalent to summation over all possible many electron states which can be constructed from the given single-electron basis.

\section{Results for $\mathrm{Yb}, \mathrm{Lu}$ and $\mathrm{Hf}$}

First, we carry out the calculations for "reference" atoms $\mathrm{Yb}, \mathrm{Lu}$ and $\mathrm{Hf}$ which have the closest electronic 
TABLE IV: Energies $\left(E, \mathrm{~cm}^{-1}\right)$ and $g$-factors of the lowest states of Nobelium. Non-relativistic values of $g$-factors $\left(g_{\mathrm{nr}}\right)$ are given by Eq. (20). Comparison with theoretical results presented by Borschevsky et al. [9] and Liu et al. [10].

\begin{tabular}{|c|c|c|c|c|c|c|c|}
\hline \multirow[t]{2}{*}{ Conf. } & \multirow{2}{*}{ Term } & \multicolumn{2}{|c|}{ Energy } & \multicolumn{2}{|c|}{ Energy } & \multicolumn{2}{|c|}{ g-factors } \\
\hline & & Present & +Lamb & Ref. [9] & Ref. [10] & Present & $\mathrm{nr}$ \\
\hline $7 s 2$ & ${ }^{1} S_{0}$ & 0 & 0 & 0 & 0 & 0 & 0 \\
\hline \multirow[t]{3}{*}{$7 s 7 p$} & ${ }^{3} P_{0}$ & 19682 & 19567 & 18879 & 19798 & 0 & 0 \\
\hline & ${ }^{3} P_{1}$ & 21156 & 21042 & 20454 & 21329 & 1.4577 & 1.500 \\
\hline & ${ }^{3} P_{2}$ & 26225 & 26113 & 25374 & 26186 & 1.4998 & 1.500 \\
\hline $7 s 7 p$ & ${ }^{1} P_{1}$ & 30304 & 30203 & 30056 & 30069 & 1.0409 & 1.000 \\
\hline \multirow[t]{3}{*}{$7 s 6 d$} & ${ }^{3} D_{1}$ & 28587 & 28436 & 28338 & & 0.5000 & 0.500 \\
\hline & ${ }^{3} D_{2}$ & 29098 & 28942 & 28778 & & 1.1606 & 1.167 \\
\hline & ${ }^{3} D_{3}$ & 30322 & 30183 & 29897 & & 1.3332 & 1.333 \\
\hline $7 s 6 d$ & ${ }^{1} D_{2}$ & 33657 & 33504 & 32892 & & 1.0071 & 1.000 \\
\hline $7 s 8 s$ & ${ }^{3} S_{1}$ & 35815 & 35731 & 35092 & & 1.9994 & 2.000 \\
\hline $7 s 8 s$ & ${ }^{1} S_{0}$ & 37444 & 37360 & 36538 & & 0.0000 & 0.000 \\
\hline \multirow[t]{3}{*}{$7 s 8 p$} & ${ }^{3} P_{0}$ & 41365 & 41266 & 40576 & & 0.0000 & 0.000 \\
\hline & ${ }^{3} P_{1}$ & 41481 & 41382 & 40692 & & 1.4083 & 1.500 \\
\hline & ${ }^{3} P_{2}$ & 42582 & 42484 & 42837 & & 1.4999 & 1.500 \\
\hline $7 s 8 p$ & ${ }^{1} P_{1}$ & 43011 & 42910 & 42285 & & 1.0917 & 1.000 \\
\hline \multirow[t]{3}{*}{$7 s 7 d$} & ${ }^{3} D_{1}$ & 43522 & 43422 & 42726 & & 0.5002 & 0.500 \\
\hline & ${ }^{3} D_{2}$ & 43581 & 43481 & 42758 & & 1.1452 & 1.167 \\
\hline & ${ }^{3} D_{3}$ & 43830 & 43730 & 43033 & & 1.3333 & 1.333 \\
\hline $7 s 7 d$ & ${ }^{1} D_{2}$ & 44099 & 43999 & 43079 & & 1.0216 & 1.000 \\
\hline $7 s 9 s$ & ${ }^{3} S_{1}$ & 44894 & 44794 & 44247 & & 1.9994 & 2.000 \\
\hline \multirow[t]{3}{*}{$7 s 6 f$} & ${ }^{3} F_{2}$ & 46795 & 46695 & & & 0.6669 & 0.667 \\
\hline & ${ }^{3} F_{3}$ & 46788 & 46688 & & & 1.0072 & 1.083 \\
\hline & ${ }^{3} F_{4}$ & 46810 & 46710 & & & 1.2500 & 1.250 \\
\hline $7 s 6 f$ & ${ }^{1} F_{3}$ & 46806 & 46706 & & & 1.0762 & 1.000 \\
\hline
\end{tabular}

structure to the superheavy No, Lr, and Rf, respectively. Since the energies of $\mathrm{Yb}, \mathrm{Lu}$, and $\mathrm{Hf}$ are known experimentally, such calculation provides the benchmark test of our method. Further comparison of correlation corrections in "reference" and superheavy atoms allow to predict the accuracy of our approach for superheavy elements.

Our calculated energy levels of $\mathrm{Yb}$, Lu and Hf are compared with experiment in Tables II II] and [II respectively. Yb energy levels have been presented in Ref. 28]. Comparison shows that relative theoretical error in excitation energies is rather similar for $\mathrm{Yb}, \mathrm{Lu}$, and $\mathrm{Hf}$, with somewhat better accuracy for two-electron $\mathrm{Yb}$.

The difference with experiment is $0.1-1.6 \%$ for $\mathrm{Yb}$ with the exception of the singlet $6 s 6 p{ }^{1} P_{1}$ and $6 s 7 p{ }^{1} P_{1}$ states, where it is $3-3.5 \%$. The lower accuracy of the singlet states arises when there is significant difference in the position of the triplet and singlet energy levels, such as in ${ }^{1} P_{1}$ and ${ }^{3} P_{1}$ case. It is $1.1-2.2 \%$ for $\mathrm{Lu}$, with the exception of $6 s^{2} 6 p$ states where it is $3-5 \%$.

A common problem of the CI calculation with four va- lence electrons is rapid increase of the number of manyelectron basis states with the increase in the number of valence electrons usually leading to omitting configurations which correspond to multiple excitations of valence electrons from the ground state to high-lying states. This helps to reduce the CI matrix to a manageable size but leads to deterioration of the accuracy of the calculations. However, we were able to saturate the four-electron CI space by carrying out several very large CI calculations with diffident types of excitations, then selecting the configuration with the largest weights from each of the runs, and combining them to produce nearly complete configuration space. Comparing results with increasing number of selected important configurations produced estimated uncertainty due to configuration space of less than $50 \mathrm{~cm}^{-1}$ for most states. As a result, we do not observe significant deterioration of results between $\mathrm{Lu}$ and $\mathrm{Hf}$. The difference with experiment is $0.1-2.9 \%$ for $\mathrm{Hf}$ with the exception of the singlet ${ }^{1} D_{2}$ and ${ }^{1} G_{4}$ states, where it is $4 \%$ and $8 \%$, respectively.

We also present the values of calculated and nonrelativistic magnetic $g$-factors in Tables [I, [I] [II] Nonrelativistic (nr) values are given by

$$
g_{\mathrm{nr}}=1+\frac{J(J+1)-L(L+1)+S(S+1)}{2 J(J+1)},
$$

where $J$ is total angular momentum of the atom, $L$ is its angular momentum and $S$ is the spin $(\mathbf{J}=\mathbf{L}+\mathbf{S})$. The $g$-factors are useful for identification of the states.

\section{RESULTS AND DISCUSSION}

\section{A. Energy levels of No, Lr and Rf and estimates of their uncertainties}

Calculated energy levels and magnetic $g$-factors for No, $\mathrm{Lr}$ and $\mathrm{Rf}$ are presented in Tables IV, V] and VI together with the results of earlier calculations [8 17]. We observe good agreement between the theoretical results for most of the states. We compare No results with and without the QED correction in Table IV] The value of the QED correction is of the order of $100 \mathrm{~cm}^{-1}$ for most of the states while maximum value of the correction is slightly larger than $200 \mathrm{~cm}^{-1}$. This is smaller than the uncertanty due to correlations (see the difference between theory and experiment for $\mathrm{Yb}, \mathrm{Lu}$ and $\mathrm{Hf}$ in Tables [1. [I. III). Therfore, we do not include QED corrections for $\mathrm{Lr}$ and Rf.

The accuracy of the calculations was discussed in previous section for the case of $\mathrm{Yb}, \mathrm{Lu}$ and $\mathrm{Hf}$ atoms. However, since relativistic and correlations effects are larger in the superheavy elements it is reasonable to assume that the uncertainty for No, Lr and Rf are slightly larger than for $\mathrm{Yb}, \mathrm{Lu}$ and $\mathrm{Hf}$. We verified that the contribution of the Breit interaction to the energy levels in Tables [- VI is small in all cases (generally less than $100 \mathrm{~cm}^{-1}$ ). 
TABLE V: Calculated energies $\left(E, \mathrm{~cm}^{-1}\right)$ and $g$-factors of the lowest states of lawrencium. Comparison with other calculations. Non-relativistic values of $g$-factors $\left(g_{\mathrm{nr}}\right)$ are given by (20).

\begin{tabular}{|c|c|c|c|c|c|c|c|c|c|}
\hline \multirow[t]{2}{*}{ Config. } & \multirow[t]{2}{*}{ Term } & \multirow[t]{2}{*}{$J$} & \multicolumn{3}{|c|}{ Present work } & \multicolumn{4}{|c|}{ Other energy } \\
\hline & & & Energy & $g$ & $g_{\mathrm{nr}}$ & Ref. [12] & Ref. [13] & Ref. [14] & Ref. [15] \\
\hline \multirow[t]{2}{*}{$7 s^{2} 7 p$} & ${ }^{2} \mathrm{P}^{o}$ & $1 / 2$ & 0 & 0.6652 & 0.6666 & 0 & 0 & 0 & 0 \\
\hline & & $3 / 2$ & 8495 & 1.3333 & 1.3333 & 8273 & 8935 & 8138 & 8389 \\
\hline \multirow[t]{2}{*}{$7 s^{2} 6 d$} & ${ }^{2} \mathrm{D}$ & $3 / 2$ & 1555 & 0.8002 & 0.8000 & 1263 & 1127 & 1331 & 1408 \\
\hline & & $5 / 2$ & 5423 & 1.2001 & 1.2000 & 5062 & & 4187 & 5082 \\
\hline \multirow[t]{4}{*}{$7 s 7 p 6 d$} & ${ }^{4} \mathrm{~F}^{o}$ & $3 / 2$ & 21288 & 0.4803 & 0.4000 & & & 20886 & \\
\hline & & $5 / 2$ & 23530 & 1.0668 & 1.0286 & & & 23155 & \\
\hline & & $7 / 2$ & & & 1.2381 & & & 27276 & \\
\hline & & $9 / 2$ & & & 1.3333 & & & 32775 & \\
\hline $7 s^{2} 8 s$ & ${ }^{2} \mathrm{~S}$ & $1 / 2$ & 20253 & 2.0163 & 2.0000 & & & 20405 & 20131 \\
\hline \multirow[t]{2}{*}{$7 s^{2} 8 p$} & ${ }^{2} \mathrm{P}^{o}$ & $1 / 2$ & 25912 & 0.6161 & 0.6666 & & & & 26104 \\
\hline & & $3 / 2$ & 27079 & 1.3174 & 1.3333 & & & & 27491 \\
\hline $7 s 6 d^{2}$ & ${ }^{4} \mathrm{P}$ & $1 / 2$ & 25409 & 2.4737 & 2.6667 & & & & \\
\hline $7 s 6 d^{2}$ & & $3 / 2$ & 26327 & 1.5286 & 1.7333 & & & & \\
\hline $7 s 6 d^{2}$ & & $5 / 2$ & 27397 & 1.3148 & 1.6000 & & & & \\
\hline
\end{tabular}

TABLE VI: Energies $\left(E, \mathrm{~cm}^{-1}\right)$ and $g$-factors of the lowest states of Rutherfordium. Non-relativistic values of $g$-factors $\left(g_{\mathrm{nr}}\right)$ are given by (20). Comparison with results by Kaldor [16].

\begin{tabular}{|c|c|c|c|c|c|}
\hline \multirow[t]{2}{*}{ Conf. } & \multirow[t]{2}{*}{ Term } & \multicolumn{2}{|c|}{ Energy } & \multicolumn{2}{|c|}{ g-factors } \\
\hline & & Present & [16] & Present & $\mathrm{nr}$ \\
\hline \multirow[t]{3}{*}{$7 s^{2} 6 d^{2}$} & ${ }^{3} F_{2}$ & 0 & 0 & 0.7291 & 0.667 \\
\hline & ${ }^{3} F_{3}$ & 4904 & 4855 & 1.0834 & 1.083 \\
\hline & ${ }^{3} F_{4}$ & 8625 & 7542 & 1.2062 & 1.250 \\
\hline \multirow[t]{3}{*}{$7 s^{2} 7 p 6 d$} & ${ }^{3} F_{2}$ & 2547 & 2210 & 0.7869 & 0.667 \\
\hline & ${ }^{3} F_{3}$ & 11390 & 11905 & 1.1041 & 1.083 \\
\hline & $3 F_{4}$ & 20477 & & 1.2489 & 1.250 \\
\hline \multirow[t]{3}{*}{$7 s^{2} 6 d^{2}$} & ${ }^{3} P_{0}$ & 5034 & & 0.0 & 0.0 \\
\hline & ${ }^{3} P_{1}$ & 8348 & 8776 & 1.4996 & 1.500 \\
\hline & ${ }^{3} P_{2}$ & 7398 & 7542 & 1.1853 & 1.500 \\
\hline \multirow[t]{3}{*}{$7 s^{2} 7 p 6 d$} & ${ }^{3} D_{1}$ & 8288 & 8373 & 0.6794 & 0.500 \\
\hline & ${ }^{3} D_{2}$ & 11273 & 10905 & 1.1493 & 1.167 \\
\hline & ${ }^{3} D_{3}$ & 18029 & & 1.2016 & 1.333 \\
\hline $7 s^{2} 6 d^{2}$ & ${ }^{1} D_{2}$ & 13630 & & 1.2531 & 1.000 \\
\hline $7 s^{2} 6 d^{2}$ & ${ }^{1} G_{4}$ & 14476 & & 1.0439 & 1.000 \\
\hline $7 s^{2} 7 p 6 d$ & ${ }^{1} D_{2}$ & 14403 & & 1.0650 & 1.000 \\
\hline $7 s^{2} 6 d^{2}$ & ${ }^{1} S_{0}$ & 18679 & & 0.0 & 0.0 \\
\hline $7 s^{2} 7 p 6 d$ & ${ }^{1} F_{3}$ & 24634 & & 1.1077 & 1.000 \\
\hline \multirow[t]{3}{*}{$7 s 6 d^{3}$} & ${ }^{5} F_{1}$ & 21552 & & 0.0962 & 0.000 \\
\hline & ${ }^{5} F_{2}$ & 23079 & & 1.0289 & 1.000 \\
\hline & ${ }^{5} F_{3}$ & 25432 & & 1.2475 & 1.250 \\
\hline \multirow[t]{2}{*}{$7 s^{2} 7 p 6 d$} & ${ }^{3} P_{1}$ & 16551 & & 1.2712 & 1.500 \\
\hline & ${ }^{3} P_{2}$ & 21480 & & 1.2267 & 1.500 \\
\hline \multirow[t]{2}{*}{$7 s 6 d^{2} 7 p$} & ${ }^{5} G_{2}$ & 20347 & & 0.5067 & 0.333 \\
\hline & ${ }^{5} G_{3}$ & 23325 & & 0.9523 & 0.917 \\
\hline
\end{tabular}

To estimate the accuracy of our values, we directly compare the correlation effects in $\mathrm{Lu}$ and $\mathrm{Lr}$, since these dominate the uncertainty of the calculations. We carry out an additional calculations for both atoms using a combination of the configuration interaction and second-order many-body perturbation theory (CI+MBPT) methods 32]. In this approach, the $\Sigma_{1}$ and $\Sigma_{2}$ of the effective Hamiltonian are build using the second-order perturbation theory instead of the coupledcluster method. The difference of the CI+MBPT and $\mathrm{CI}+$ all-order values gives the estimate of the third and higher-order corrections. We note that $\mathrm{Lu}$ and $\mathrm{Lr}$ have different types of the ground state configuration, $6 s^{2} 5 d{ }^{2} D_{3 / 2}$ and $7 s^{2} 7 p^{2} P_{1 / 2}$. Therefore, we first directly compare the higher-order correlation contributions to the three-electron removal energies of $\mathrm{Lu}$ and $\mathrm{Lr}$ which are given in Table VII. Columns CI+MBPT and CI+all give trivalent removal energies calculated in the respective approximations. The difference of these values give the estimate of the higher-order correlation correction given in column labeled "Diff". Last column labelled "Diff*" gives the higher-order correlations relative to the corresponding ground states. We find that while the energies are similar for $\mathrm{Lu}$ and $\mathrm{Lr}$, the higher-order correlation corrections significantly increases from Lu (2000 $\left.3200 \mathrm{~cm}^{-1}\right)$ to $\operatorname{Lr}\left(3800-6300 \mathrm{~cm}^{-1}\right)$. However, we observe that the correlation increases for all of the states and when the ground state values are subtracted out, the remaining higher-order corrections, listed in the last column of Table VII are very similar for Lu and Lr. Only for the three states, $7 s^{2} 6 d^{2} D_{3 / 2}$ and $7 s 6 d^{2}{ }^{4} D_{3 / 2,5 / 2}$, the remaining contributions are larger than for Lu cases, which may result in somewhat lower accuracy for there states.

Therefore, we expect $1-2 \%$ accuracy of the energy levels in No, $1-3 \%$ in Lr, and $2-5 \%$ for Rf for most of 
TABLE VII: Comparison of higher-order $\left(\mathrm{III}^{+}\right)$correlation contributions to three-electron removal energies of $\mathrm{Lu}$ and Lr. Columns CI+MBPT and CI+sll give removal energies calculated in the respective approximations. The difference of these values give the estimate of the higher-order correlation correction given in column labeled Diff. Last column labelled Diff* give the higher-order correlation relative to the corresponding ground states.

\begin{tabular}{|c|c|c|c|c|c|}
\hline Atom & Level & $\mathrm{CI}+\mathrm{MBPT}$ & $\mathrm{CI}+$ all & Diff & Diff* \\
\hline \multirow[t]{9}{*}{$\mathrm{Lu}$} & $6 s^{2} 5 d^{2} D_{3 / 2}$ & 328791 & 325983 & -2808 & 0 \\
\hline & $6 s^{2} 5 d^{2} D_{5 / 2}$ & 326610 & 323981 & -2629 & 179 \\
\hline & $5 d^{2} 6 s^{4} F_{3 / 2}$ & 309931 & 306716 & -3215 & -406 \\
\hline & $5 d^{2} 6 s^{4} F_{5 / 2}$ & 309307 & 306166 & -3141 & -333 \\
\hline & $5 d^{2} 6 s^{4} F_{7 / 2}$ & 308356 & 305330 & -3026 & -217 \\
\hline & $5 d^{2} 6 s^{4} F_{9 / 2}$ & 307222 & 304323 & -2898 & -90 \\
\hline & $6 s^{2} 6 p^{2} P_{1 / 2}$ & 324182 & 322187 & -1996 & 812 \\
\hline & $6 s^{2} 6 p^{2} P_{3 / 2}$ & 320859 & 318866 & -1993 & 815 \\
\hline & $5 d 6 s 6 p^{4} F_{3 / 2}$ & 310376 & 308268 & -2107 & 701 \\
\hline \multirow[t]{12}{*}{ Lr } & $7 s^{2} 6 d^{2} D_{3 / 2}$ & 337828 & 331718 & -6110 & -1422 \\
\hline & $7 s^{2} 6 d^{2} D_{5 / 2}$ & 333287 & 327872 & -5415 & -726 \\
\hline & $7 s^{2} 8 s^{2} S_{1 / 2}$ & 318449 & 313137 & -5311 & -623 \\
\hline & $7 s 6 d^{2}{ }^{4} P_{1 / 2}$ & 311742 & 307921 & -3821 & 867 \\
\hline & $7 s 6 d^{2}{ }^{4} P_{3 / 2}$ & 313159 & 306879 & -6280 & -1592 \\
\hline & $7 s 6 d^{2}{ }^{4} P_{5 / 2}$ & 311830 & 305825 & -6005 & -1317 \\
\hline & $7 s^{2} 7 p^{2} P_{1 / 2}$ & 338055 & 333366 & -4688 & 0 \\
\hline & $7 s^{2} 7 p^{2} P_{3 / 2}$ & 329645 & 324877 & -4768 & -80 \\
\hline & $7 s 7 p 6 d^{4} F_{3 / 2}$ & 316309 & 311992 & -4318 & 371 \\
\hline & $7 s 7 p 6 d^{4} F_{5 / 2}$ & 314099 & 309752 & -4348 & 341 \\
\hline & $7 s^{2} 8 p^{2} P_{1 / 2}$ & 312532 & 307454 & -5079 & -391 \\
\hline & $7 s^{2} 8 p^{2} p_{3 / 2}$ & 311250 & 306266 & -4984 & -296 \\
\hline
\end{tabular}

the states presented here.

\section{B. Ionization potentials}

Calculations in the $V^{N-M}$ approximation are very similar for a neutral atom, negative and positive ions [23]. The number of valence electrons is the only parameter in the effective CI Hamiltonian (11) which changes while moving from a neutral atom to an ion or from one ion to another. All other terms, including Coulomb potential created by core electrons and correlation operator $\hat{\Sigma}$ remain the same. Removing one electron from a neutral atom and comparing the energy of resulting ground state with the energy of the ground state of neutral atom give first ionization potential of the atom. Removing one more electron leads to second ionization potential, etc. This process can be repeated until all valence electrons are removed. The number of ionization potentials which can be calculated this way is limited by the number of valence electrons. To illustrate the accuracy of the calculations we calculate ionization potentials for $\mathrm{Yb}, \mathrm{Lu}$ and Hf and compare them with experiment. The results are presented in Table VIII. Then in the same table we present ionization potentials for No, Lr and Rf.
TABLE VIII: Calculated ground state energies $\left(E_{M}\right)$ of $\mathrm{Yb}$, $\mathrm{Lu}, \mathrm{Hf}, \mathrm{No}, \mathrm{Lr}$ and Rf neutral atoms and positive ions. $M$ is the number of valence electrons. The difference $\Delta E=$ $E_{M-1}-E_{M}$ gives the ionization potential.

\begin{tabular}{llllcrr}
\hline \hline $\begin{array}{l}\text { Atom } \\
\text { /Ion }\end{array}$ & $\begin{array}{l}\text { Configu- } \\
\text { ration }\end{array}$ & Term & $M$ & $\begin{array}{c}E_{M} \\
{[\text { a.u. }]}\end{array}$ & $\begin{array}{r}\Delta E \\
{\left[\mathrm{~cm}^{-1}\right]}\end{array}$ & $\begin{array}{r}\text { Expt. } \\
{\left[\mathrm{cm}^{-1}\right]}\end{array}$ \\
\hline Yb I & $6 s^{2}$ & ${ }^{1} \mathrm{~S}_{0}$ & 2 & -0.68232 & 50768 & 50443 \\
Yb II & $6 s$ & ${ }^{2} \mathrm{~S}_{1 / 2}$ & 1 & -0.45101 & 98985 & 98207 \\
Lu I & $6 s^{2} 5 d$ & ${ }^{2} \mathrm{D}_{3 / 2}$ & 3 & -1.48938 & 43289 & 43763 \\
Lu II & $6 s^{2}$ & ${ }^{1} \mathrm{~S}_{0}$ & 2 & -1.29215 & 113323 & 112000 \\
Lu III & $6 s$ & ${ }^{2} \mathrm{~S}_{1 / 2}$ & 1 & -0.77581 & 170270 & 169014 \\
Hf I & $6 s^{2} 5 d^{2}$ & ${ }^{3} \mathrm{~F}_{0}$ & 4 & -2.83907 & 53431 & 55048 \\
Hf II & $6 s^{2} 5 d$ & ${ }^{2} \mathrm{D}_{3 / 2}$ & 3 & -2.59562 & 126748 & 120000 \\
Hf III & $6 s^{2}$ & ${ }^{1} \mathrm{~S}_{0}$ & 2 & -2.01811 & 190885 & 187800 \\
Hf IV & $6 s$ & ${ }^{2} \mathrm{~S}_{1 / 2}$ & 1 & -1.14837 & 252037 & 269150 \\
No I & $7 s^{2}$ & ${ }^{1} \mathrm{~S}_{0}$ & 2 & -0.72799 & 54390 & \\
No II & $7 s$ & ${ }^{2} \mathrm{~S}_{1 / 2}$ & 1 & -0.48018 & 105387 & \\
Lr I & $7 s^{2} 7 p$ & ${ }^{2} \mathrm{P}_{1 / 2}^{o}$ & 3 & -1.52543 & 39801 & \\
Lr II & $7 s^{2}$ & ${ }^{1} \mathrm{~S}_{0}$ & 2 & -1.34408 & 118058 & \\
Lr III & $7 s$ & ${ }^{2} \mathrm{~S}_{1 / 2}$ & 1 & -0.80617 & 176934 & \\
Rf I & $7 s{ }^{2} 6 d^{2}$ & ${ }^{3} \mathrm{~F}_{0}$ & 4 & -2.79968 & 46067 & \\
Rf II & $7 s^{2} 5 d$ & ${ }^{2} \mathrm{D}_{3 / 2}$ & 3 & -2.58979 & 116925 & \\
Rf III & $7 s^{2}$ & ${ }^{1} \mathrm{~S}_{0}$ & 2 & -2.05704 & 193246 & \\
Rf IV & $7 s$ & ${ }^{2} \mathrm{~S}_{1 / 2}$ & 1 & -1.17654 & 258220 & \\
\hline \hline
\end{tabular}

${ }^{a}$ Ref. [29].

\section{Static polarizabilities}

Results of calculations of static polarizabilities of $\mathrm{Yb}$, $\mathrm{Lu}, \mathrm{Hf}, \mathrm{No}, \mathrm{Lr}$ and $\mathrm{Rf}$ are presented in Table IX. $\mathrm{CI}+\mathrm{MBPT}$ and $\mathrm{CI}+$ all-order results are listed in columns labelled "MBPT" and "All-order", respectively. The calculations are done as described in section IIC, The result for ytterbium agrees precisely with our previous calculations [28, 33, 34], with experimental constrain presented in Ref. [35], and with most of other accurate calculations (see, e. g. review [36]), the results for lutetium and hafnium agree well with the calculations of Doolen [37]. Estimation of accuracy is based on comparison of the results obtained with the use of different approaches, including comparison with experiment for ytterbium, and on the sensitivity of the results to variation of the parameters of the computational procedure. The theoretical uncertainty presented in the parentheses is on the level of $5 \%$ for $\mathrm{Yb}$, Lu and $\mathrm{Hf}$ (see Table IX). We expect similar uncertainty for No and Rf. Lawrencium represents a special case due to anomalously small energy interval between ground ${ }^{2} \mathrm{P}_{1 / 2}$ state and first excited ${ }^{2} \mathrm{D}_{3 / 2}$ state. Note that there is an inversion of the order of these states in $\mathrm{Lr}$ as compared to its lighter analog Lu. The inversion is due to relativistic effects [11 15]. Because of small value of this energy interval it is very sensitive to the correlations. Different treatment of correlations lead to significantly different values of the interval (see TableV). This in turn leads to large uncertainty in the value of the polarizabilities of both states of Lr. 
TABLE IX: Ground state scalar $\alpha_{0}$ and tensor $\alpha_{2}$ polarizabilities of $\mathrm{Yb}, \mathrm{Lu}, \mathrm{Hf}, \mathrm{No}, \mathrm{Lr}$, and Rf. CI+MBPT and $\mathrm{CI}+$ all-order results are listed in columns labelled "MBPT" and "All-order", respectively. Last column presents the values of $\alpha_{0}$ from other sources. All numbers are in atomic units. To convert them into $10^{-24} \mathrm{~cm}^{3}$ one should divide the numbers by 6.749 .

\begin{tabular}{|c|c|c|c|c|c|}
\hline \multirow{2}{*}{$\begin{array}{l}\text { Atom/ } \\
\text { State }\end{array}$} & \multicolumn{2}{|c|}{$\alpha_{0}$} & \multicolumn{2}{|c|}{$\alpha_{2}$} & \multirow{2}{*}{$\begin{array}{c}\alpha_{0} \\
\text { Other }\end{array}$} \\
\hline & MBPT & All-order & MBPT & All-order & \\
\hline $\mathrm{Yb}{ }^{1} \mathrm{~S}_{0}$ & $141(6)^{a}$ & $141(2)^{b}$ & 0 & 0 & $139.3(4.9)^{c}$ \\
\hline $\mathrm{Lu}{ }^{2} \mathrm{D}_{3 / 2}$ & $137(7)$ & 145 & $-15(1)$ & -22 & $148^{d}$ \\
\hline Hf ${ }^{3} \mathrm{~F}_{2}$ & $103(5)$ & 97 & $-0.41(2)$ & -0.92 & $109^{d}$ \\
\hline $\mathrm{No}^{1} \mathrm{~S}_{0}$ & $112(6)$ & 110 & 0 & 0 & \\
\hline $\operatorname{Lr}{ }^{2} \mathrm{P}_{1 / 2}$ & $320(80)$ & 323 & 0 & 0 & \\
\hline $\operatorname{Lr} \quad{ }^{2} \mathrm{D}_{3 / 2}$ & $-12(25)$ & -12 & $120(25)$ & 119 & \\
\hline $\mathrm{Rf}{ }^{3} \mathrm{~F}_{2}$ & $107(5)$ & 115 & $2.3(4)$ & 8.9 & \\
\hline
\end{tabular}

${ }^{a}$ Agrees precisely with our previous calculations, Ref. [33, 34]. ${ }^{b}$ Ref. [28].

${ }^{c}$ Experimental constrain, Ref. 35].

${ }^{d}$ Relativistic linear response calculations by G. D. Doolen, unpublished, cited from Ref. 37].

The value of the electric dipole transition amplitude between $7 p_{1 / 2}$ and $6 d_{3 / 2}$ states of $\operatorname{Lr}$ in the calculations is given by

$$
\left\langle 7 s^{2} 7 p_{1 / 2}|| \mathbf{D} \| 7 s^{2} 6 d_{3 / 2}\right\rangle=2.02 \text { a.u. }
$$

This allows us to separate the contribution due to this transition from the rest of the sum in (12) and (13) and present polarizabilities in the form

$$
\begin{aligned}
& \alpha_{0}\left(7 p_{1 / 2}\right)=126+1.35 / \Delta E, \\
& \alpha_{0}\left(6 d_{3 / 2}\right)=67-0.677 / \Delta E, \\
& \alpha_{2}\left(6 d_{3 / 2}\right)=26+0.677 / \Delta E,
\end{aligned}
$$

where all values are in atomic units and $\Delta E=E\left(6 d_{3 / 2}\right)-$ $E\left(7 p_{1 / 2}\right)$. Sensitivity of the polarizabilities to the value of this energy interval is the main source of uncertainty. The uncertainty assigned to the polarizabilities of lawrencium (Table IX. MBPT column) are based on the variation of the energy interval in different calculations (Table $(\mathrm{V})$. The uncertainties for other atoms are smaller due to absence of the resonance contribution. The most accurate values are those obtained in the all-order calculations while the difference between all-order and MBPT results can serve as en estimation of theoretical uncertainty.

Knowing the value of the electric dipole transition amplitude (21) allows us to calculate lifetime of the $6 d_{3 / 2}$ state. It is $0.23 \mathrm{~ms}$ if we take the energy interval to be our theoretical value of $1555 \mathrm{~cm}^{-1}$ (see Table $\mathrm{V}$ ). This is a long-lived metastable state. Since lawrencium atoms are not found in nature but produced on accelerators they can probably be produced in either of the $7 p_{1 / 2}$ or $6 d_{3 / 2}$ states. The interaction with environment is very different for $\mathrm{Lr}$ atoms in these two states. It is isotropic for the atoms in the $7 p_{1 / 2}$ state and strongly anisotropic for atoms in the $6 d_{3 / 2}$ state. In the later case, the polarizability is dominated by the tensor term. The total value is positive $(\alpha \approx 100$ a.u. $)$ for the case when total atomic angular momentum is parallel to the electric field $(|M|=J)$ and it is negative $(\alpha \approx-160$ a.u.) for the case when total atomic angular momentum is perpendicular to the electric field $(M=0)$.

\section{CONCLUSION}

Energy levels for lowest states of superheavy elements nobelium, lawrencium and rutherfordium as well as first few ionization potentials and static polarizabilities have been calculated using the combination of the configuration interaction with the all-order single-double methods. The accuracy of the calculations are controlled by performing similar calculations for lighter analogs of the elements, ytterbium, lutecium, and hafnium. These calculations provide benchmark data, critically evaluated for their accuracy, for future experimental studies.

\section{Acknowledgments}

The work was supported in part by the Australian Research Council and by USA NSF Grant No. PHY1212442 .
[1] V. Pershina, Radiochim. Acta 99, 459 (2011).

[2] V. Pershina, Hem. Rev. 113, 1237 (2013).

[3] H. Backe, F. P. Heßberger, M. Sewtz, and A. Türler, Eur. Phys. J. D 45, 3 (2007).

[4] T. H. Dinh, V. A. Dzuba, V. V. Flambaum, and J. S. M. Ginges, Phys. Rev. A 78, 022507 (2008).

[5] T. H. Dinh, V. A. Dzuba, V. V. Flambaum, and J. S. M. Ginges, Phys. Rev. A 78, 054501 (2008).

[6] T. H. Dinh, V. A. Dzuba, and V. V. Flambaum, Phys. Rev. A 78, 062502 (2008).

[7] V. A. Dzuba, Phys. Rev. A 88, 042516 (2013).
[8] S. Fritzsche, Eur. Phys. J. D 33, 15 (2005).

[9] A. Borschevsky, E. Eliav, M. J. Vilkas, Y. Ishikawa, and U. Kaldor, Phys. Rev. A 75, 042514 (2007).

[10] Y. Liu, R. Hutton, and Y. Zou, Phys. Rev. A 76, 062503 (2007).

[11] J.-P. Desclaux and B. Frike, J. de Phys. 41, 943 (1980).

[12] E. Eliav, U. Kaldor, and Y. Ishikawa, Phys. Rev. A 52, 291 (1995).

[13] Yu Zou and C. Froese Fischer, Phys. Rev. Lett. 88, 183001 (2002).

[14] S. Fritzsche, C. Z. Dong, F. Koike, and A. Uvarov, Eur. 
Phys. J. D 45, 107 (2007).

[15] A. Borschevsky, E. Eliav, M. J. Vilkas, Y. Ishikawa, and U. Kaldor, Eur. Phys. J. D 45, 115 (2007).

[16] E. Eliav, U. Kaldor, and Y. Ishikawa, Phys. Rev. Lett. 74, 1079 (1995).

[17] W. C. Martin and Jack Sugar, Phys. Rev. A 53, 1911 (1996).

[18] M. S. Safronova, M. G. Kozlov, W. R. Johnson, and Dansha Jiang, Phys. Rev. A 80, 012516 (2009).

[19] M. S. Safronova, M. G. Kozlov, and C. W. Clark, Phys. Rev. Lett. 107, 143006 (2011).

[20] S. G. Porsev, M. S. Safronova, and M. G. Kozlov, Phys. Rev. Lett. 108, 173001 (2012).

[21] M. S. Safronova, S. G. Porsev, and C. W. Clark, Phys. Rev. Lett. 109, 230802 (2012).

[22] M. S. Safronova, V. A. Dzuba, V. V. Flambaum, U. I. Safronova, S. G. Porsev, M. G. Kozlov, arXiv:1405.4271, submitted to Phys. Rev. Lett. (2014).

[23] V. A. Dzuba, Phys. Rev. A 71, 032512 (2005).

[24] W. R. Johnson and J. Sapirstein, Phys. Rev. Lett. 57, 1126 (1986).

[25] V. A. Dzuba, C. Harabati, W. R. Johnson, and M. S. Safronova, Phys. Rev. A 63, 044103 (2001).

[26] V. A. Dzuba, V. V. Flambaum, and M. S. Safronova, Phys. Rev. A 73, 022112 (2006).
[27] V. V. Flambaum and J. S. M. Ginges, Phys. Rev. A 72, 052115 (2005).

[28] S. G. Porsev, M. S. Safronova, A. Derevianko, and C. W. Clark, Phys. Rev. A 89, 012711 (2014).

[29] A. Kramida, Yu. Ralchenko, J. Reader, and NIST ASD Team (2012). NIST Atomic Spectra Database (ver. 5.0), [Online]. Available: http://physics.nist.gov/asd [2013, August 19]. National Institute of Standards and Technology, Gaithersburg, MD.

[30] J. E. Sansonetti and W. C. Martin, J. Phys. Chem. Ref. Data 34, 1559 (2005).

[31] A. Dalgarno and J. T. Lewis, Proc. R. Soc. London, Ser. A 223, 70 (1955).

[32] V. A. Dzuba, V. V. Flambaum, and M. G. Kozlov, Phys. Rev. A 54, 3948 (1996).

[33] V. A. Dzuba and A. Derevianko, J. Phys. B 43, 074011 (2010).

[34] V. A. Dzuba, A. Kozlov, and V. V. Flambaum, arXiv:1403.5002 (2014).

[35] K. Beloy, Phys. Rev. A 86, 022521 (2012).

[36] J. Mitroy, M. S. Safronova, and C. W. Clark, J. Phys. B 43, 202001 (2010).

[37] T. M. Miller, in CRC Handbook of Chemistry and Physics, Ed. D. R. Lide (CRC Press New York, 2002). 